\title{
Studies and observations on the health effects of drinking electrolyzed-reduced alkaline water
}

\author{
B. Rubik \\ Institute for Frontier Science, Oakland, California, USA
}

\begin{abstract}
Municipal drinking water, prefiltered and treated by partial electrolysis, followed by collecting the cathodic water that is alkaline ( $\mathrm{pH} 8.5$ to 9.5), shows a high negative oxidative reductive potential (ORP) $(-150$ to $-250 \mathrm{mV})$ compared to untreated tap water $(+150 \mathrm{mV})$ as well as smaller molecular clusters. A growing body of literature indicates beneficial effects from drinking this water by patients with diabetes and kidney disease, with improved outcomes and fewer medical complications. Additional studies suggest that this water increases the activity of a key detoxifying enzyme in the body, superoxide dismutase, which is central to protecting against free radical damage both in aging and chronic degenerative disease. Recent published studies on the health benefits of this drinking water are summarized. Evidence from live blood analysis from a case study suggests that drinking reduced alkaline water reduces blood cell stickiness, aggregation, and early clotting. Results suggest that long term consumption of this water slows the effects of aging and may improve the peripheral circulation; serve as an adjunct therapy for diabetes and kidney disorders; and help prevent cardiovascular and other chronic diseases.
\end{abstract}

Keywords: ionized water, electrolyzed alkaline water, reduced water, microwater, microstructured water, energized water, red blood cells, erythrocytes, fibrin, cardiovascular health, diabetes, inflammation, cancer.

\section{Introduction}

Water, which constitutes over $70 \%$ of the human body, is involved in virtually every function of life. It is an essential but often underrated necessity that is involved in most biochemical reactions; a constituent of the bodily fluids blood, lymph, cerebrospinal fluid, saliva and other digestive fluids; joint 
lubrication; detoxification; and maintaining the blood pressure. Yet it is far more than just a constituent. A new science of water is emerging in which the structure and dynamics of water is much more complex than was previously thought. Water is a complex dynamic liquid sensitive and responsive to its environment. We are now in the midst of a paradigm shift in which liquid water is seen as an active agent rather than a passive constituent or universal solvent of life. For example, water and living systems are equally sensitive to a single quantum of magnetic flux [1]. Water shows long-range ordering features around cell membranes, where is it more like an epitaxial liquid crystal with distinct properties in those regions that radically distinguish it from bulk water [2] while chemically still remaining $\mathrm{H}_{2} \mathrm{O}$. It used to be thought that water was passive, "dancing to the tune of biomolecules", but now, water is considered the "matrix of life."

The greatest physiological change with aging is not within our biomolecules, but in the loss of water from the body. The body of a young infant consists of over $80 \%$ water, but that of a person over 70 years old is typically comprised of less than $60 \%$ water.

There are many concerns about drinking water quality worldwide today. Many people choose to drink commercially bottled water for various reasons, but its healthfulness is questionable. Some device manufacturers claim that water treated by a vortex, electromagnetic fields, or other physical means to "energize" it, is more healthful than untreated waters and may also slow the aging process. In this paper, we review published evidence for the impact on health of drinking a particular type of water called "electrolyzed-reduced" water and show observations from the blood.

\section{Background and literature review}

Alkaline mineral water with a relatively high negative oxidative reductive potential (ORP $=-150$ to -300 millivolts) and a $\mathrm{pH}$ over 8.0 is characteristic of natural mountain streams and certain deep wells. Aside from these natural sources, where can one find water with these properties? If this natural water were to be bottled, it would lose its high negative ORP and may react with plastic bottles that contain phthalates, toxic carcinogens. Nonetheless, one can generate water with these properties at point-of-use by using a device called an ionizer. This is a commercially available water treatment system for the home that first filters the water through a multi-stage filter to remove chlorine, chloramine, and other contaminants, and then performs partial electrolysis of the filtered water over platinum-coated titanium electrodes with a DC electric field. This yields 2 fractions: oxidized acidic water from the anode, and reduced alkaline water from the cathode. The reduced alkaline water appears to match most closely natural mountain spring water at the source in its physical properties and taste. This water is the subject of this paper. It has been called by various names: reduced, electrolyzed-reduced, and alkaline ionized water, to name a few. In this paper, we refer to it as ERW, "electrolyzed-reduced water." The ERW fraction retains the alkaline ionic minerals from the tap water, 
including calcium and magnesium, which are important minerals for health, has a high negative ORP, an alkaline $\mathrm{pH}$, a low level of dissolved oxygen, and is microstructured, with 5 to 6 molecules of water per cluster. It also has a lower surface tension than the starting tap water, which makes it a better solvent and may improve hydration.

Most of the studies on ERW have been conducted in Japan, Korea, and China. Not all of the research reports have been translated into English. Peer-reviewed studies from various laboratories worldwide show that ERW, with its high negative ORP, scavenges free radical chemical species, protecting from oxidative damage. This, along with its alkalinity and microstructure, yield numerous health benefits. In addition, clinical reports, with or without controlled studies supporting them, further suggest that ERW produces declines in blood sugar levels in diabetic patients; improvements in peripheral circulation in diabetic gangrene; improvements in intestinal flora; declines in uric acid levels in patients with gout; improvements in liver function tests in hepatic disorders; improvements in gastroduodenal ulcer with prevention of recurrences; improved hydration and fluid replacement; and improvements in blood pressure in the case of either hypertension or hypotension. Here we summarize some of the key findings from the peer-reviewed literature on humans and other biological systems.

\subsection{Active reducing agent and protection against oxidative stress}

Shirahata et al. studied the properties of ERW and reported that it showed a superoxide dismutase-like activity in protecting against oxidative damage, alleviating oxidative damage of DNA molecules and other species in vitro [3]. This antioxidant effect of ERW has been verified [4]. The nature of the reducing species (antioxidant) in ERW has been proposed to be active hydrogen and/or molecular hydrogen, but it is not the same as ordinary hydrogen gas and remains unresolved [5, 6]. One group reports that ERW contains both atomic and molecular hydrogen [7]. ERW prevented oxidative cleavage of proteins and also stimulated the activity of free radical scavenger, ascorbic acid [8]. Rats, upon drinking ERW for just one week, showed significantly reduced amounts of peroxidized lipid in their urine, suggesting reduced oxidative stress in the rats [9]. These studies document that ERW has strong antioxidant activity. Antioxidant activity is important to protect cells and biomolecules from the toxic effects of oxidative damage associated with reactive oxygen species such as superoxide radicals that are associated with the biochemistry of inflammation and implicated as underlying factors in chronic disease.

\subsection{Prolonged lifespan in nematodes and mice}

It is well accepted that oxidative damage culminates in many of the maladies of aging, so that a drinking water with antioxidant activity might be helpful to mitigate the ravages of aging and possibly slow it down. G. Fernandes at the University of Texas reported that various strains of mice fed ERW from birth lived 20 to $50 \%$ longer. He also found reduced serum peroxide levels in these 
mice compared to control mice fed tap water [10]. Landis and Tower showed that enhanced activity of superoxide dismutase, as has been demonstrated by various investigators using ERW, can reduce oxidative damage and extend life span [11].

A study on ERW used in the aqueous medium of the nematode (worm), C. Elegans in laboratory cultures showed that it significantly extended its lifespan, which has been interpreted to be at least in part due to the reactive oxygen species (ROS)-scavenging action of ERW [12].

\subsection{Studies on kidney disease and use of hemodialysis}

In end-stage kidney-diseased patients on dialysis, ERW appears to have a beneficial effect on reduction of hemodialysis-induced oxidative stress. Huang et al. studied the reactive oxygen species in the plasma of these patients and found that ERW diminished hemodialysis-enhanced peroxide levels, and minimized oxidized and inflammatory markers (C-reactive protein and interleukin-6) after 1 month of drinking ERW. These findings suggest that cardiovascular complications (stroke and heart attack) in these kidney dialysis patients might be prevented by ERW [13].

Another study investigated use of ERW directly in the hemodialysis process of 8 kidney patients, and found that the viability of patients' polymorphonuclear leukocytes was better preserved [14].

\subsection{Studies on diabetes and blood glucose levels}

Reactive oxygen species (ROS), such as superoxide and other free radical oxygen species, are known to cause reduction of glucose update by inhibiting the insulin-signaling pathway in cultured cells. Therefore, the scavenging of ROS is important to the control of diabetes. ERW scavenged intracellular ROS and stimulated glucose uptake in the presence or absence of insulin in rat L6 skeletal muscle cells and mouse 3T3/L1 adipocytes. This insulin-like activity of ERW was inhibited by wortmannin, a specific inhibitor of PI-3 kinase, a key molecule in insulin signalling pathways. ERW protected insulin responsive cells from sugar toxicity and improved the damaged sugar tolerance of type II diabetes model mice. This suggests that ERW may improve the status of those with insulin-independent diabetes mellitus [15].

Oxidative stress is produced under diabetic conditions and involved in progression of pancreatic beta-cell dysfunction. ERW in diabetic mice improved islet beta-cell function, resulting in increased release of circulating insulin and improved insulin sensitivity in both type I and type II diabetes [16, 17]. In a study on Otsuka Long-Evans Tokushima Fatty (OLETF) rats, ERW given to one group showed significantly lower blood glucose levels than controls given tap water. Moreover, blood levels of triglycerides and total cholesterol also decreased in the rats fed ERW [18].

A study on 411 type II diabetes patients whose average age was 71.5 years, who drank natural reduced water from the Nordenau Spring in Germany, up to 2 liters per day over 6 days, showed that $186(45 \%)$ responded positively, with 
reduced blood glucose, improved cholesterol, LDL, HDL, and serum creatinine levels. $70.6 \%$ of a random sample of 136 of the patients also showed a decrease in blood ROS [19].

Recent bioelectrical impedance analysis studies showed that diabetics have a lower ratio of intracellular water (ICW) to extracellular water (ECW). 336 typeII diabetics were recruited in a randomized, double-blind trial. The subjects received $250 \mathrm{ml}$ of ERW or distilled water twice daily for 4 weeks. Results show that ERW consumption improved cell water distribution (ICW/ECW), basal metabolism rate, and cell capacitance during the 4 -week period. The authors speculate that the relatively small size of the water molecule clusters in ERW may underlie the beneficial findings of improved cell structure and function [20].

\subsection{Stimulation of anaerobic microflora in the human gut}

The high negative ORP of ERW favors the growth of key anaerobic bacteria in the human gut that are important for normal intestinal microflora, health of the colon, and optimum nutrition [21].

\subsection{Lack of toxicity in microbes, cells, and animals}

ERW used up to a concentration of $100 \%$ in the Ames Test with Salmonella typhimurium did not show any bacterial mutations, either in the presence or absence of rat liver for exogenous metabolic activation. Similarly, ERW did not induce any chromosome aberrations in Chinese hamster lung fibroblast cells with or without rat liver, for up to 24 hours. Rats administered ERW at a dose of $20 \mathrm{~mL} / \mathrm{kg} /$ day for 28 days via intragastric infusion did not show any clinical symptoms or toxic changes. These results demonstrate the expected safety for a $60 \mathrm{~kg}$ human to drink at least 1.2L/day of ERW [22].

Developing animals are the most sensitive to biological agents and are often used in studies to investigate toxicity. Thus, ERW was given to pregnant and also lactating rats to look for any effects. Development of rat fetuses and offspring were normal, and ERW increased the weight of the animals over controls. ERW was also found to have positive biological effects on postnatal growth. Moreover, postnatal morphological development was also accelerated [23]. No significant difference in milk yield or suckled milk volume was noted. It is suspected that the water-hydrated calcium cations transferred to the fetus through the placenta and to the offspring through the milk, might be the cause of the increased body weight, since calcium plays a key role in skeletal formation [24].

\subsection{Inhibition of cancer but not normal cells}

It is known that tumor cells produce ROS more abundantly than normal cells. It is also well known that antioxidants can inhibit tumor cell proliferation, which indicates an important role of ROS in mediating the loss of growth control. Human tongue carcinoma cells were shown to be significantly inhibited for 
either colony formation or colony sizes by ERW in cell cultures without inhibition to normal human tongue epithelial cells. ERW also caused growth inhibition, cell degeneration, and inhibition of invasion to human fibrosarcoma cells HT-1080. These studies suggest that ERW may help prevent tumor progression and invasion [25].

In vitro examination of leukemia cells (HL-60) treated with ERW showed enhanced mitochondrial damage and cell apoptosis. However, normal peripheral blood mononuclear cells showed no cytotoxic effect from ERW [26]. ERW also suppressed the growth rate of cancer cells transplanted into mice, demonstrating anti-cancer effects in vivo.

\subsection{Protection of liver from toxic agents}

Mice with carbon tetrachloride-induced liver damage given ERW showed significantly lowered serum levels of hepatic enzyme markers and increased activities of superoxide dismutase and other key detoxifying enzymes. The effects of ERW were similar to silymarin, an extract from milk thistle well known for its hepato-protective properties. Results suggest that ERW may be used to protect the liver against toxins that induce oxidative damage [27].

\subsection{Conclusions from the literature review}

These studies show impressive health benefits in humans and other biological systems from consumption of ERW over a very short time, and without any toxic effects observed. Clearly, ERW is a useful adjunct for treating ROS-associated diseases, including diabetes, kidney disease, cancer, and cardiovascular disease. In addition, due to its anti-aging effects in scavenging oxygen free radicals, ERW appears to be an excellent choice for regular water consumption, although its antioxidant activity is unstable upon storage. Nonetheless, it is easily produced from tap water at point-of-use.

\section{Observations from live blood analysis}

The blood is the most easily monitored tissue that can show rapid changes that correlate with health and disease. We have observed that persons drinking ERW show exceptionally clean biological terrains as monitored by live blood analysis.

Live blood analysis is the visual examination of a small droplet of fresh capillary blood typically taken from the fingertip, put onto a glass slide, and immediately observed under a high-powered light microscope equipped with a dark-field condenser. This method offers a visual perspective of the blood cells and plasma at high magnification enhanced by modern optical techniques. It provides an assessment of the ecology of the blood, the "biological terrain". Live blood analysis is used clinically to look for the malaria and Lyme disease parasites. Here we discuss it as a tool to assess blood cell stickiness, clumping, and coagulation and clotting processes, which are related to the activation of the inflammatory cascade. 
A microphotograph from live blood analysis is shown in Figure 1. This is a photograph of normal healthy blood of a fasted female, age 37 . The red blood cells (RBCs) are seen as single, free, round cells. Only a few platelet aggregates are seen in the plasma as grey areas. No RBC stickiness and no other clotting factors are found throughout the blood sample.

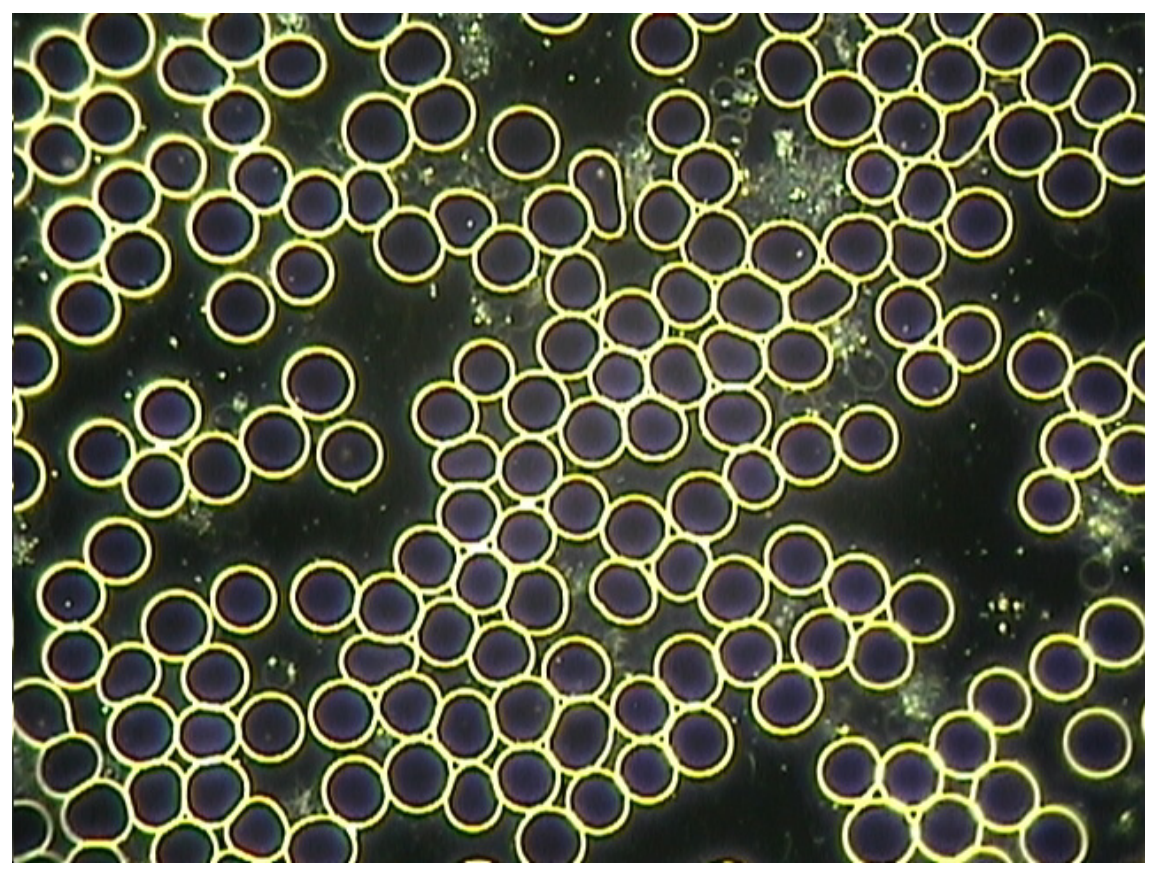

Figure 1: $\quad$ Normal healthy blood from female, age 37.

By contrast, Figure 2 shows the blood of a male, 65 years old. This blood is also typical of that found in many elderly persons. The RBCs are sticky and tightly clumped together in rouleau (rolls of coins seen on edge). Fibrin (white threads) is present, indicating that blood coagulation and clotting have been activated. This is the picture of systemic inflammation.

Peripheral circulation was also impaired for this subject, because only single RBC can move freely through the smallest capillaries. Poor circulation in the extremities is a common complaint of the elderly.

Following this test, the subject, M, age 65, drank 1 to 1.5 liters/day of ERW from an ionizer for 6 months but made no other changes in diet or lifestyle. Figure 3 shows the blood from the same person after 6 months. The RBC stickiness, aggregation, and clotting factors are no longer present. It is particularly striking to see this change in an older person's blood. Although this is a single case presented here, numerous other cases have been observed as well. 


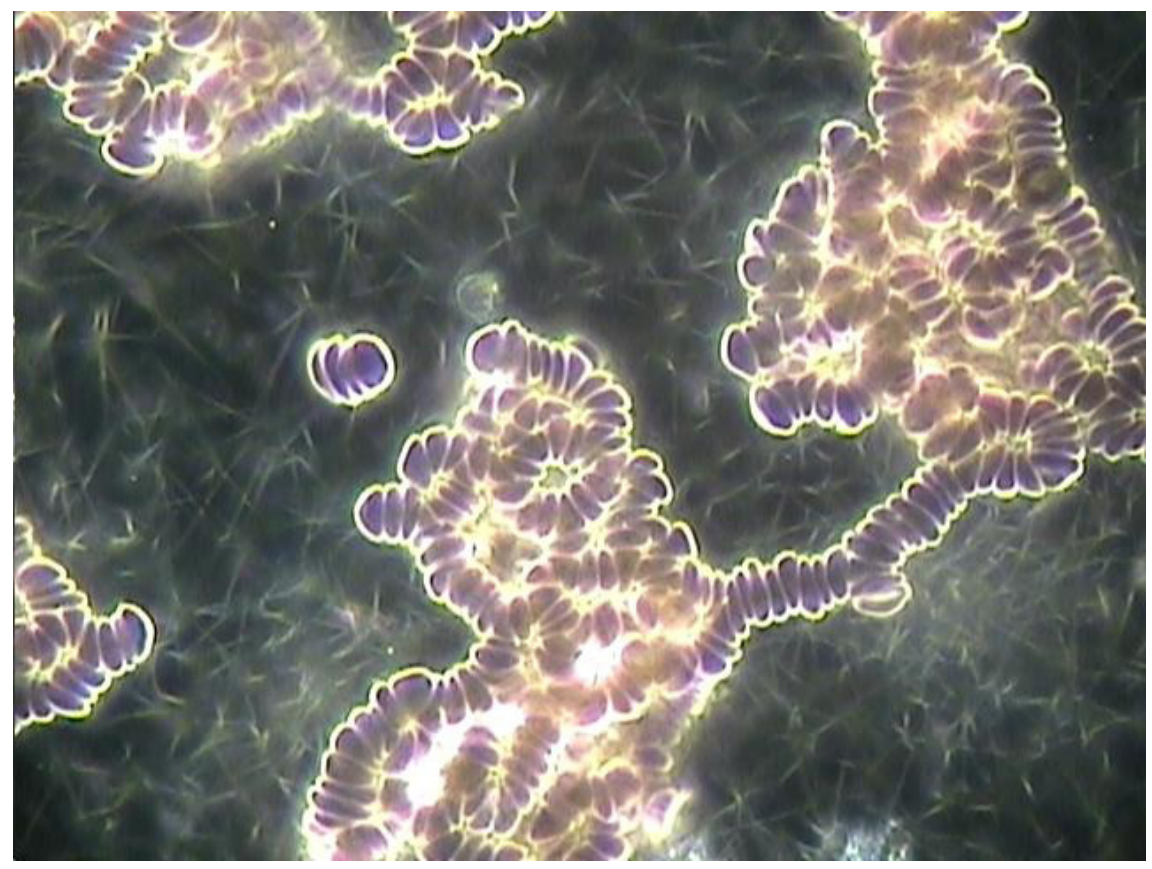

Figure 2: Unhealthy blood from male, 65, showing blood congestion and clotting.

\section{Conclusions}

Chronic inflammation is considered to be one of the main underlying factors of virtually all of the chronic degenerative diseases, including cancer, cardiovascular, and autoimmune diseases. From observing changes in the biological terrain apparently due to consumption of ERW, it appears that ERW may be a useful intervention to mitigate activation of the clotting and inflammatory pathways. Long-term consumption of ERW may improve the blood circulation and possibly help prevent the chronic diseases of our times.

Acid-alkaline balance is another key to health and wellness [28]. Metabolism of food leads to acid wastes, yet the biological terrain needs to be alkaline, $\mathrm{pH}$ 7.2-7.4. Drinking alkaline water such as ERW can contribute to neutralizing acid waste and maintaining proper $\mathrm{pH}$ balance in the body.

In conclusion, a growing body of scientific and clinical literature shows increasing support for ERW as a "functional" drinking water that scavenges free radicals, diminishes systemic inflammation, and is a useful adjunct for treating ROS-associated diseases, including diabetes, kidney disease, cancer, and cardiovascular disease. From observations of the blood, it appears to mitigate early blood clotting and systemic inflammation seen as sticky, aggregated RBCs and fibrin. Collectively, this evidence points to ERW as a healthy drinking water 


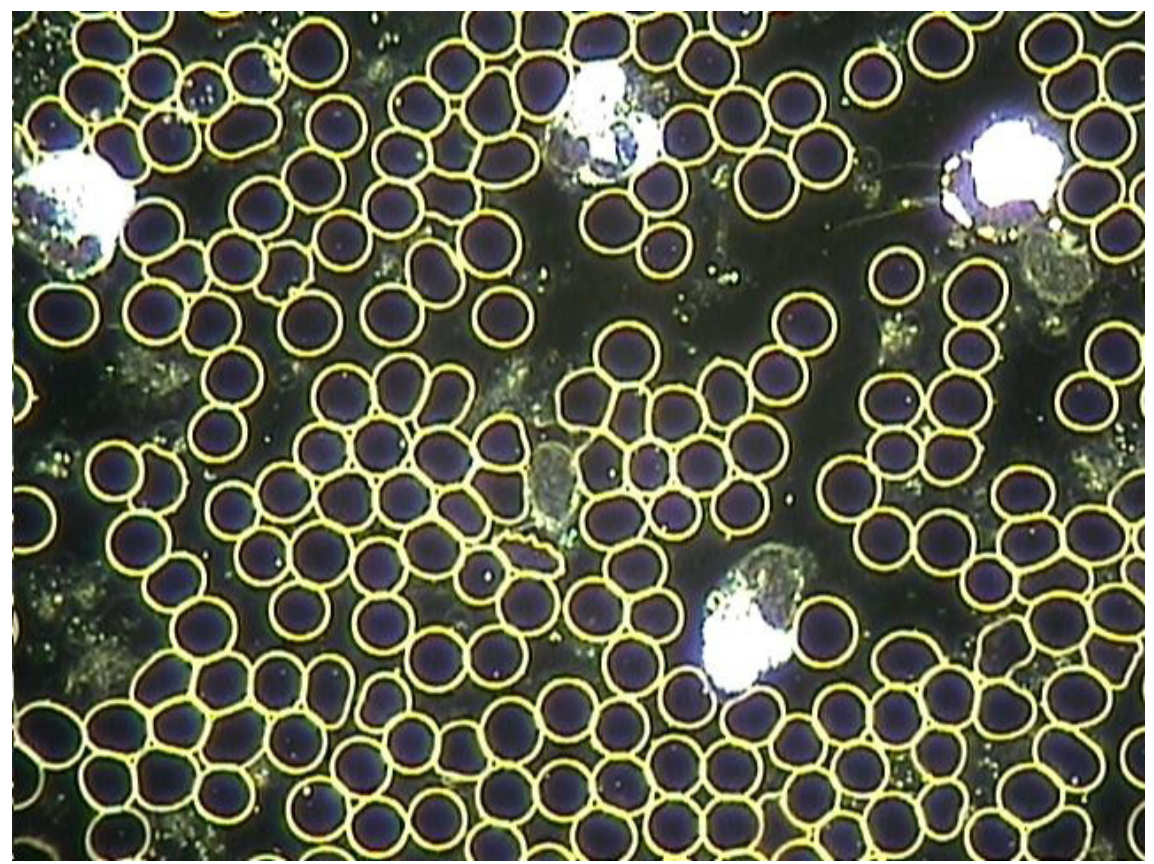

Figure 3: Blood of same male, 65, after drinking 1.5 liters ERW for 6 months. This biological terrain is normal and healthy. Several white blood cells are also seen here.

that slow down the effects of aging. More research is recommended to further study its anti-aging and anti-inflammatory effects.

\section{References}

[1] Smith, C.W., Quanta and coherence effects in water and living systems. Journal of Alternative and Complementary Medicine, 10(1), pp. 69-79, 2004.

[2] Zheng, J.-M., Pollack, G.H., Solute exclusion and potential distribution near hydrophilic surfaces. In: Water and the Cell, eds Pollack, G.H., Cameron, I.L., Wheatley, D.N., Springer: Dordrecht, the Netherlands, pp. 165-174, 2006.

[3] Shirahata, S., Kabayama, S., Nakano, M., Miura, T., Kusumoto, K., Gotoh, M., Hayashi, H., Otsubo, K., Morisawa, Y. and Katakura, Y., Electrolyzedreduced water scavenges active oxygen species and protects DNA damage. Biochem. Biophys. Res. Commun., 234, pp. 269-274, 1997.

[4] Hanaoka, K., Sun, D., Lawrence, R., Kamitani, Y., Fernandes, G., The mechanism of the enhanced antioxidant effects against superoxide anion radicals of reduced water produced by electrolysis. Biophysical Chemistry 107, pp. 71-82, 2004. 
[5] Hiraoka, A., Takemoto, M., Suzuki, T., Shinohara, A., Chiba, M., Shirae, M., Yoshimura, Y., Studies on the properties and real existence of aqueous solution systems that are assumed to have antioxidant activities by the action of "active hydrogen." Journal of Health Science 50(5), pp. 456-465, 2004.

[6] Hanaoka, K. Antioxidant effects of reduced water produced by electrolysis of sodium chloride solutions. Journal of Applied Electrochemistry 31, pp. 1307-1313, 2001.

[7] Nakanishi, K., Hamasaki, T, Nakamura, T, Abe, M, and Teruya, K. Growth suppression of HL70 and L6 cells by atomic hydrogen. Animal Cell Technology: Basic \& Applied Aspects 16, pp. 323-325, 2009.

[8] Lee, M.Y., Kim, Y.K., Ryoo, K.K., Lee, Y.B., Park, E.J. Electrolyzedreduced water protects against oxidative damage to DNA, RNA, and protein. Applied Biochemistry and Biotechnology 135(2), pp. 133-144, 2006.

[9] Yanagihara, T., Arai, K., Miyamae, K., Sato, B., Shudo, T., Yamada, M., Aoyama, M., Electrolyzed reduced water for drinking elicits an antioxidant effect: a feeding test with rats. Bioscience, Biotechnology, and Biochemistry 69(10), pp. 1985-1987, 2005

[10] Fernandes, G., unpublished presentation, conference hosted by Proton Laboratories on electrolyzed reduced water, Alameda, CA, 2000.

[11] Landis, G.N., Tower, J., Superoxide dismutase, evolution, and life span regulation. Mechanisms of Aging and Development 126(3), pp. 365-79, 2005.

[12] Yan, H., Tian, H., Hamasaki, T., Abe, M., Nakamichi, N. Electrolyzed reduced water prolongs Caenorhabditis elegans' lifespan. Animal Cell Technology: Basic and Applied Aspects 16, pp. 289-293.

[13] Huang, K.C., Lee, K.T., Chien, C.T., Reduced hemodialysis-induced oxidative stress in end-stage renal disease patients by electrolyzed reduced water. Kidney International 64(2), pp. 704-714, 2003.

[14] Nakayama, M., Kabayama, S., Nakano, H., Zhu, W.J., Terawaki, H., Nakayama, K., Katoh, K., Satoh, T., Ito, S. Biological effects of electrolyzed water in hemodialysis. Nephron Clinical Practice 112, pp. 9$15,2009$.

[15] Oda, M., Kusumota, K., Teruya, T., Hara, T., Maki, t., Kabayama, S., Katakura, Y., Otsubo, K., Morisawa, S., Hayashi, H. Electrolyzed and natural reduced water exhibit insulin-like activity on glucose uptake into muscle cells and adipocytes. Animal Cell Technology: Products from Cells, Cells as Products, Proc of the 16th ESACT Meeting, April 25-29, 1999, eds A. Bernard, B. Griffiths, W. Noe, F. Wurm. Kluwer Academic Publishers: New York, Chapter VII, pp. 425-427, 2002.

[16] Kim, M.J., Kim, H.K. Anti-diabetic effects of electrolyzed reduced water in streptozotocin-induced and genetic diabetic mice. Life Sciences 79, 228891, 2006.

[17] Kim, M.J., Jung, K.H., Uhm, Y.K, Leem, K.H., Kim, H.K. Preservative effect of electrolyzed reduced water on pancreatic beta-cell mass in diabetic 
$\mathrm{db} / \mathrm{db}$ mice. Biological and Pharmaceutical Bulletin 30(2), pp. 234-236, 2007.

[18] Jin, D., Ryu, S.H., Kim, H.W., Yang, E.J., Lim, S.J., Ryang, Y.S., Chung, C.H., Park, S.K., Lee, K.J. Anti-diabetic effect of alkaline-reduced water on OLETF rats. Bioscience, Biotechnology, and Biochemistry 70(1), pp. 3137, 2006.

[19] Gadek, Z., Hamasaki, T., Shirahata, S., "Nordenau Phenomenon" application of natural reduced water to therapy. Animal Cell Technology: Basic and Applied Aspects, 15, pp. 265-271, 2009.

[20] Wang, Z.Y., Zhou, Z.C., Zhu, K.N., Wang, X., Pan, J.G., Lorenzen, L.H., Zhou, M.C., Microclustered water and hydration. Asia Pacific Journal of Clinical Nutrition 13(Suppl.), S128.

[21] Vorobjeva, N.V., Selective stimulation of the growth of anaerobic microflora in the human intestinal tract by electrolyzed reducing water. Medical Hypotheses 64(3), pp. 543-546, 2005.

[22] Saitoh, Y., Harata, Y., Mizukashi, F., Nakajima, M., Miwa, N. Biological safety of neutral-pH hydrogen-enriched electrolyzed water upon mutagenicity, genotoxicity, and subchronic oral toxicity. Toxicology and Industrial Health 26(4), pp. 203-216, 2010.

[23] Watanabe, T., Effect of alkaline ionized water on reproduction in gestational and lactational rats. Journal of Toxicology Science 20(2), pp. 135-142, 1995.

[24] Watanabe T., Pan, I., Fukuda, Y., Murasugi, E., Kamata, H., Uwatoko, K. Influences of alkaline ionized water on milk yield, body weight of offspring, and perinatal dam in rats. Journal of Toxicology Science 23(5), pp. 365-71, 1998.

[25] Saitoh, Y., Okayasu, H., Xiao, L., Harata, Y., Miwa, N. Neutral pH hydrogen-enriched electrolyzed water achieves tumor-preferential clonal growth inhibition over normal cells and tumor invasion inhibition concurrently with intracellular oxidant repression. Oncology Research 17, pp. 247-255, 2008.

[26] Tsai, C.F., Hsu, Y.W., Chen, W.K., Ho, Y.C., Lu, F.J. Enhanced induction of mitochondrial damage and apoptosis in human leukemia HL-60 cells due to electrolyzed-reduced water and glutathione. Bioscience, Biotechnology, and Biochemistry 73(2), pp. 280-287, 2009.

[27] Tsai, C.F., Hsu, Y.W., Chen, W.K., Chang, W.H., Yen, C.C., Ho, Y.C., Lu, F.J. Hepatoprotective effect of electrolyzed reduced water against carbon tetrachloride-induced liver damage in mice. Food and Chemical Toxicology 47, pp. 2031-2036, 2009.

[28] Minich, D.M., Bland, J.S. Acid-alkaline balance: role In chronic disease and detoxification. Alternative Therapies 13(4), pp. 62-65, 2007. 